\title{
Influence of Parameters on Flexoelectro-Optic Effect in Cholesteric Liquid Crystals
}

\author{
M. BUCZKOWSKA* \\ Institute of Physics, Lodz University of Technology, Wólczańska 219, 90-924 Eódź, Poland
}

Received: 01.07.2021 \& Accepted: 18.08.2021

Doi: 10.12693/APhysPolA.140.258

*e-mail: mariola.buczkowska@p.lodz.pl

\begin{abstract}
The deformations of a cholesteric liquid crystal layer with a helical axis parallel to the layer plane are investigated numerically. When such a layer, called the Uniform Lying Helix texture, is placed between crossed polarizers, then the system gives an interesting linear electro-optic effect provided that the cholesteric possesses flexoelectric properties. Namely, the electric field perpendicular to the layer plane rotates the optical axis of the liquid crystal around the layer normal by an angle nearly proportional to the field strength. As a result, the optical transmission of the system is controlled by the bias voltage. The present study is devoted to numerical simulations of the Uniform Lying Helix layers. The director distributions in the layers were calculated by means of minimizing the free energy of the system. The voltage dependence of the rotation angle and of the pitch, occurring at various system parameters, was determined. The influence of parameters on efficiency of the flexoelectro-optic effect expressed by the derivative of the rotation angle with respect to bias voltage was studied. The results showed that high efficiency is favoured by long pitch, small sum of bend and splay elastic constants, large difference between splay and bend flexoelectric coefficients, zero dielectric anisotropy and thin layer which confirmed the earlier theoretical predictions.
\end{abstract}

topics: flexoelectro-optic effect, cholesterics, director deformations

\section{Introduction}

Optical properties of cholesteric liquid crystals result from a relation between light wavelength $\lambda$ and their spatial periodicity manifested by pitch $p$. In particular, if $p$ is sufficiently smaller than $\lambda$, a cholesteric behaves like a uniaxial medium with an optical axis parallel to the helical axis, i.e., perpendicular to the twisted director. This feature gives an interesting linear electro-optic effect provided that the cholesteric possesses flexoelectric properties [1]. The flexoelectro-optic effect occurs in cholesteric liquid crystals confined in layers with homeotropic boundary conditions. The optical axis is then parallel to the layer plane creating the socalled Uniform Lying Helix (ULH) texture [2-5]. If the applied electric field is perpendicular to the layer, then the director and - in consequence - the optical axis rotate around the layer normal by an angle $\Phi$ proportional to the field strength $E$. If the layer of thickness $d$ is placed between crossed polarizers, the optical transmission of the system can be controlled by the bias voltage $U=E d$. In the optimum case, the zero field helical axis should make an angle of $22.5^{\circ}$ with one of the polarizers while the rotation angle $\Phi$ should vary between $-22.5^{\circ}$ and $+22.5^{\circ}$ with respect to the undistorted helical axis under the action of positive and negative voltage, respectively [6, 7]. Cholesteric materials exhibiting large rotation angles $|\Phi| \geq 45^{\circ}$ can also be used if the undeformed helical axis coincides with one of the polarizers $[8,9]$. In both configurations, the optical transmission of the system can be electrically switched between 0 and 1 .

Theoretical formulae for the rotation angle and for the pitch $p$ varying under the action of the electric field have already been derived $[1,2]$. They are given, respectively, as

$$
\begin{gathered}
\tan (\Phi)=\frac{\left(e_{11}-e_{33}\right) p_{0} E}{2 \pi\left(k_{11}-k_{33}\right)}, \\
\tan (\Phi)=\frac{\left(e_{11}-e_{33}\right) p_{0} E}{2 \pi k_{22}} \\
-\frac{k_{11}+k_{33}-2 k_{22}}{2 \pi k_{22}} \sin (\Phi),
\end{gathered}
$$

and

$$
p=p_{0} \cos (\Phi),
$$

where $k_{11}, k_{22}$ and $k_{33}$ are the splay, twist and bend elastic constants, $e_{11}$ and $e_{33}$ are the flexoelectric coefficients related to splay and bend deformations, and $p_{0}$ is the intrinsic pitch in the undistorted cholesteric. The situation when the length of the pitch varies with the change of field strength is described by (1), whereas (2) is suitable when the boundary conditions prevent any changes. 


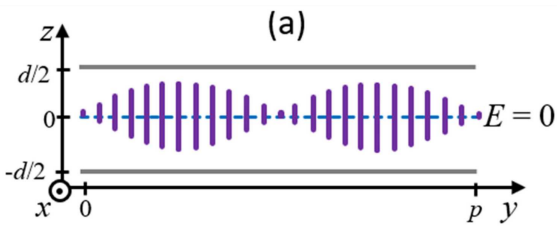

(b)

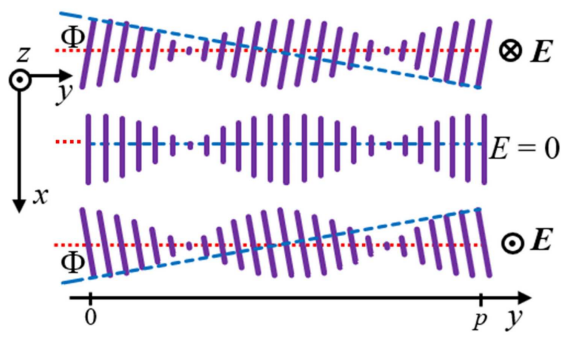

Fig. 1. Schematic presentation of the ULH structure of a chiral nematic confined in a layer of thickness $d$. The twisted structure is illustrated by means of projections of director on (a) the $y z$ plane and (b) $x y$ plane. The helical axis is indicated by a dotted line. The optical axis (a dashed line) is oriented normal to the director. Its orientations, induced by electric field of strength $E$ directed parallel and antiparallel to the $z$ axis, are presented in (b). Rotation angle $\Phi$ is indicated in both cases. In the absence of the field, the optical axis coincides with the helical axis.

In this work, electric field-induced deformations of the ULH structures were studied numerically. The aim was to verify the relationships predicted theoretically and expressed with (1)-(3). For this purpose, director distributions in the layers deformed by the electric field were calculated numerically using various sets of parameters, i.e., flexoelectric coefficients, intrinsic pitch and elastic constants. The results allowed to determine the voltage dependence of the rotation angle as well as of the effective pitch and, further, to compare them with the relationships predicted theoretically. The role of dielectric anisotropy, thickness and anchoring energy was also studied.

\section{Geometry and parameters of the system}

The geometry of the system is presented schematically in Fig. 1. The cholesteric liquid crystal layer of thickness $d$ confined between two plates playing the role of transparent electrodes parallel to the $x y$ plane of coordinate system and positioned at $z= \pm d / 2$ was considered. Such a plane-parallel layer is typical for the systems possessing an applicative potential. The lower electrode was assumed to be earthed. Homeotropic boundary conditions were imposed and they were determined by easy axes $\boldsymbol{e}_{1}=\boldsymbol{e}_{2}=[0,0,1]$ oriented normal to the lower and upper electrode, respectively. These boundary conditions ensured that the helical axis of the cholesteric was parallel to the boundary plates, producing the ULH structure. The anisotropic surface anchoring was assumed. The anisotropy was manifested by the difference between polar and azimuthal anchoring strengths, on the lower and upper electrodes, respectively [10].

The two-dimensional deformations of director distributions which lead to flexoelectro-optic effect were taken into account. It was assumed that all the physical quantities and variables describing the two-dimensional structures are dependent on two coordinates $y$ and $z$, and are constant along the $x$ axis. The director distribution $n(y, z)$ was determined by means of the polar angle $\theta(y, z)$ measured between $n$ and the $x y$ plane and by the azimuthal angle $\varphi(y, z)$ between the $x$ axis and the projection of $n$ on the $x y$ plane. First, a set of initial parameters of the layer was assumed. Their values are summarized as follows:

- intrinsic pitch $p_{0}=0.4 \mu \mathrm{m}$;

- elastic constants $k_{11}=8 \mathrm{pN}, k_{22}=4 \mathrm{pN}$, $k_{33}=12 \mathrm{pN}$;

- flexoelectric coefficients $e_{11}=10 \mathrm{pC} / \mathrm{m}$, $e_{33}=-10 \mathrm{pC} / \mathrm{m}$;

- dielectric anisotropy $\Delta \varepsilon=0$;

- polar anchoring strengths $W_{\theta_{1}}=10^{-4} \mathrm{~J} / \mathrm{m}^{2}$ and $W_{\theta_{2}}=10^{-4} \mathrm{~J} / \mathrm{m}^{2}$ on the lower and upper electrode, respectively;

- azimuthal anchoring strengths $W_{\varphi_{1}}=$ $10^{-5} \mathrm{~J} / \mathrm{m}^{2}$ and $W_{\varphi_{2}}=10^{-5} \mathrm{~J} / \mathrm{m}^{2}$ on the lower and upper electrode, respectively;

- thickness $d=2 \mu \mathrm{m}$.

Each of these parameters was varied within reasonable range while the other remained unchanged. Voltage dependence of the rotation angle $\Phi$ and of the pitch $p$ were determined for each set of parameters. The cholesteric was treated as a perfect insulator.

\section{Method}

The equilibrium structures of the director field inside the layer were determined based on minimizing the free energy per unit area of the layer. The method successfully applied in earlier works, see e.g., $[11,12]$, was used for this purpose. A segment of the layer of the width equal to the pitch was considered during the computations. The periodic boundary conditions along the $y$ axis were imposed. In the sites of the $M \times N$ regular lattice, where $M=64$ and $N=65$, we introduced the electric potential $V_{i j}$ and defined the discrete angles $\theta_{i j}$ and $\varphi_{i j}$ describing the director distribution over the cross-section of the segment. The indices $i=1, \ldots, M$ and $j=1, \ldots, N$ determined the position along the $y$ and $z$ axes, respectively. The coordinates $y=0$ and $y=p$ corresponded to $i=1$ and $i=M$ whereas $z=-d / 2$ and $z=d / 2$ were labelled by $j=1$ and $j=N$. The planes determined by $i=$ const and $j=$ const divided the 
cross-section of the segment into $(M-1) \times(N-1)$ rectangular cells. The values $\theta_{i j}, \varphi_{i j}$ and $V_{i j}$ taken from the corners of each cell gave the average angles as well as the spatial derivatives of the angles and of the potential. These values were used to calculate the total free energy counted per unit length of the segment in the $x$ direction. Then, the result was divided by actual $p$ in order to obtain the total free energy per unit area of the layer. Now, the corresponding formula on the free energy

$$
\begin{aligned}
F & =\frac{1}{2 p} \int_{0}^{p} \mathrm{~d} y\left\{\int _ { - d / 2 } ^ { d / 2 } \mathrm { d } z \left[k_{11}\left(\nabla \boldsymbol{n}^{2}\right)+k_{22}\left(\boldsymbol{n}(\nabla \times \boldsymbol{n})-\frac{2 \pi}{p_{0}}\right)^{2}+k_{33}(\boldsymbol{n} \times(\nabla \times \boldsymbol{n}))^{2}\right.\right. \\
& \left.\left.-2\left(e_{11} \boldsymbol{n} \cdot \nabla \boldsymbol{n}-e_{33} \boldsymbol{n} \times(\nabla \times \boldsymbol{n})\right) \cdot \boldsymbol{E}-\frac{1}{2} \varepsilon_{0} \varepsilon_{\perp} E^{2}-\varepsilon_{0} \Delta \varepsilon(\boldsymbol{n} \cdot \boldsymbol{E})^{2}\right]\right\} \\
& +\frac{1}{2 p} \int_{0}^{p} \mathrm{~d} y\left[W_{\phi_{1}} \cos ^{2}\left(\theta_{1}-\theta_{s_{1}}\right)+W_{\theta_{1}} \sin ^{2}\left(\theta_{1}-\theta_{s_{1}}\right)\right]\left[1-\left(\boldsymbol{n}_{1} \cdot \boldsymbol{e}_{1}\right)^{2}\right] \\
& +\frac{1}{2 p} \int_{0}^{p} \mathrm{~d} y\left[W_{\phi_{2}} \cos ^{2}\left(\theta_{2}-\theta_{s_{2}}\right)+W_{\theta_{2}} \sin ^{2}\left(\theta_{2}-\theta_{s_{2}}\right)\right]\left[1-\left(\boldsymbol{n}_{2} \cdot \boldsymbol{e}_{2}\right)^{2}\right]
\end{aligned}
$$

was based on general expressions describing elastic, dielectric and flexoelectric properties of liquid crystals and their interactions with the substrate [13]. The symbols $\boldsymbol{n}_{1}$ and $\boldsymbol{n}_{2}$ denote the directors adjacent to the lower and upper plate, respectively, $\theta_{1}$ and $\theta_{2}$ are their polar orientation angles whereas $\theta_{s_{1}}=\theta_{s_{2}}=90^{\circ}$ determine the orientation of the easy axes. This energy (4) was minimized with respect to all the angles $\theta_{i j}, \varphi_{i j}$ and pitch $p$.

Initially, the values $\theta_{i j}=\frac{2 \pi}{M}(i-1)-\frac{\pi}{2}, \varphi_{i j}=0$ and $p=p_{0}$ were introduced (according to the uniform twisted director distribution in the ULH structure), where $i=1, \ldots, M$ and $j=1, \ldots, N$. In a similar manner, the initial distribution of the electric potential corresponding to uniform electric field was assumed, $V_{i j}=U(j-1) /(N-1)$. The final set of the variables $\theta_{i j}, \varphi_{i j}$ and $p$ which approximated the real equilibrium director distribution, was calculated in the course of an iteration process. During the computations, the values of $\theta_{i j}, \varphi_{i j}$ and $p$ were varied successively by small intervals.

Now, the free energy per unit area of the layer was calculated after each change. If the new energy was lower than the previous one, the changed value of the variable was accepted and the corresponding interval was increased by a factor of about 1.5. In the opposite case, the variable remained unchanged and the interval was decreased to about 0.8 of its previous value. When the new values of angles in all sites of the lattice as well as the new value of $p$ were found, the electric potential distribution $V(y, z)$ in the layer was calculated as well. For this purpose, the Poisson equation

$$
\begin{aligned}
& \varepsilon_{0}\left[\frac{\partial V}{\partial y}\left(\frac{\partial \varepsilon_{y y}}{\partial y}+\frac{\partial \varepsilon_{y z}}{\partial z}\right)+\frac{\partial V}{\partial z}\left(\frac{\partial \varepsilon_{y z}}{\partial y}+\frac{\partial \varepsilon_{z z}}{\partial z}\right)+\varepsilon_{y y} \frac{\partial^{2} V}{\partial y^{2}}+2 \varepsilon_{y z} \frac{\partial^{2} V}{\partial y z}+\varepsilon_{z z} \frac{\partial^{2} V}{\partial z^{2}}\right] \\
& \quad-e_{11}\left(\frac{\partial n_{y}}{\partial y}+\frac{\partial n_{z}}{\partial z}\right)^{2}-e_{11} n_{y}\left(\frac{\partial^{2} n_{y}}{\partial y^{2}}+\frac{\partial^{2} n_{z}}{\partial y \partial z}\right)-e_{11} n_{z}\left(\frac{\partial^{2} n_{y}}{\partial y \partial z}+\frac{\partial^{2} n_{z}}{\partial^{2} z}\right) \\
& \quad+e_{33}\left(\frac{\partial n_{z}}{\partial y} r_{x}+n_{z} \frac{\partial r_{x}}{\partial y}-\frac{\partial n_{x}}{\partial y} r_{z}-n_{x} \frac{\partial r_{z}}{\partial y}\right)+e_{33}\left(\frac{\partial n_{x}}{\partial z} r_{y}+n_{x} \frac{\partial r_{y}}{\partial z}-\frac{\partial n_{y}}{\partial z} r_{x}-n_{y} \frac{\partial r_{x}}{\partial z}\right)=0
\end{aligned}
$$

was resolved, where $r_{x}=\partial n_{z} / \partial y-\partial n_{y} / \partial z$, $r_{y}=\partial n_{x} / \partial z$ and $r_{z}=-\partial n_{x} / \partial y$, while $n_{i}$ and $\varepsilon_{i j}$ are the components of the director and of the dielectric permittivity tensor, respectively. The boundary conditions $V\left(\frac{-d}{2}\right)=0$ and $V\left(\frac{d}{2}\right)=U$ were imposed. Thus, the values of potential in the sites of the lattice $V_{i j}$ were obtained. Thereby, a single cycle of computations came to an end and resulted in the new set of variables suitable for the next cycle. The cycles were repeated until a further reduction in the total free energy could be neglected. As a result, a state of minimum energy was obtained. 


\section{Results}

The influence of the system parameters on the voltage dependence of the rotation angle and on the effective pitch was investigated. The results presented in Figs. 2-9 were obtained using initial values of parameters, excepting specified values of parameters mentioned in the captions. Also, director distributions in the cross-section of the layer over the distance of one pitch were calculated. The typical deformation arising after the application of electric field normal to the layer had the form of patterns with the splay and bend superimposed on intrinsic twist when the director is deviated from the helix axis. Figure 2a shows an example of the undistorted director distribution in the absence of external electric field.

The intrinsic twist about the axis parallel to the layer plane is evident. In Fig. 2b, the rotation of director around normal to the layer occurring under the action of bias voltage is demonstrated. This kind of deformation was connected with the rotation of the optical axis which coincides with the helical axis.

\subsection{Rotation angle}

The rotation angle was nearly constant over the cross-section of the layer. It depended slightly on the $z$-coordinate in the vicinity of boundary plates as a consequence of homeotropic boundary conditions. In order to characterize the flexoelectro-optic effect, the voltage dependence of the angle $\Phi$ and

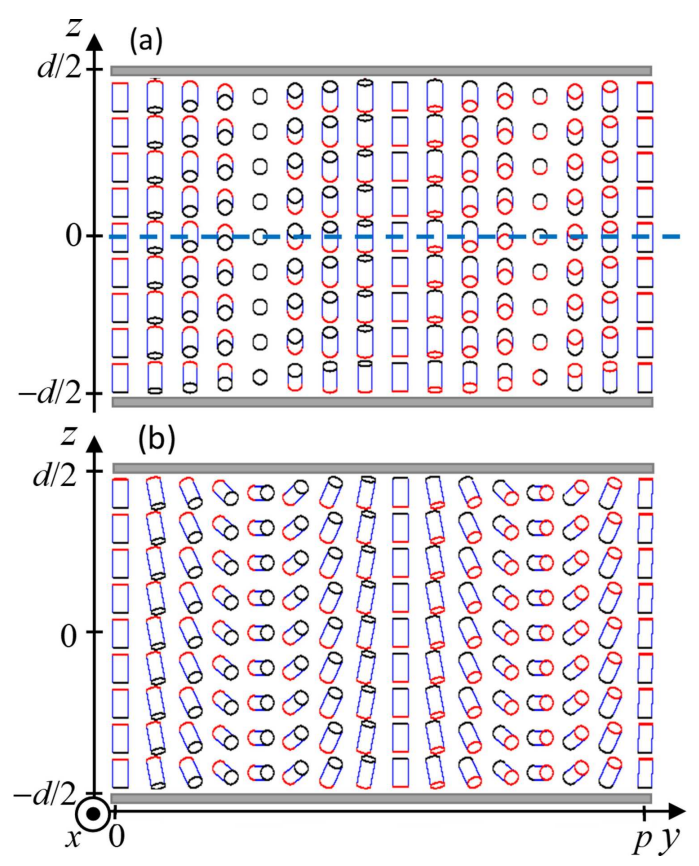

Fig. 2. Director distribution in the cross-section of the layer over the distance of one pitch. The initial set of parameters was adopted: (a) $U=0 \mathrm{~V}$, (b) $U=30 \mathrm{~V}$. A helical axis identical with the optical axis is shown in the undeformed structure.

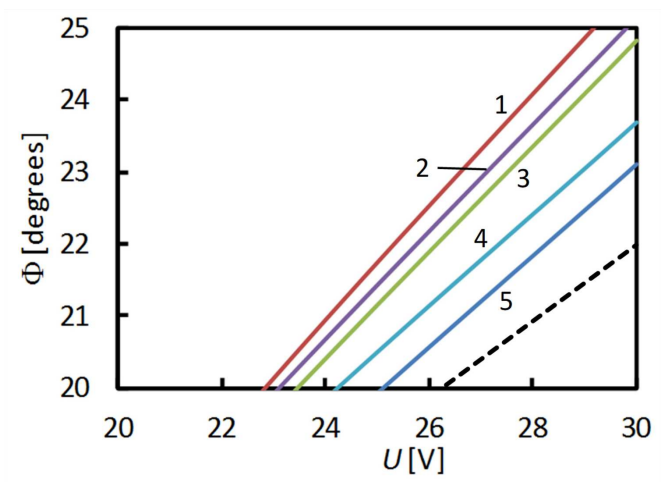

Fig. 3. Different voltage dependencies of the rotation angle $e_{11}-e_{33}=20 \mathrm{pC} / \mathrm{m}$. Detailed values of flexoelectric coefficients in $\mathrm{pC} / \mathrm{m}\left(e_{11}, e_{33}\right)$ are as follows: 1: $(10,-10) ; 2:(0,-20) ; 3:(20,0) ; 4$ : $(-10,-30) ; 5:(30,10)$. Other parameters had their initial values. A dashed line shows theoretical $\Phi(U)$ dependence obtained from (1).

of the pitch $p$ were determined. In general, the angle $\Phi$ corresponding to small deformations increased nearly proportionally to bias voltage $U$. The derivative $\mathrm{d} \Phi / \mathrm{d} U$ could serve as a measure of efficiency of the effect at a given voltage. The next sections report the consequences of variation of chosen specific parameters while the other parameters retained their initial values.

\subsubsection{Role of flexoelectric coefficients}

According to theoretical predictions expressed by (1) and (2), the rotation angle depended on the difference between flexoelectric coefficients $e_{11}-e_{33}$ and not on their particular values. This means that the following exemplary sets of flexoelectric coefficients $\left(e_{11}, e_{33}\right)$ (in $[\mathrm{pC} / \mathrm{m}]$ ) should result in similar deformations: $1:(10,-10) ; 2:(0,-20) ; 3:(20,0) ; 4$ : $(-10,-30) ; 5:(30,10)$, etc. However, the present calculations showed that individual coefficients had some weak influence even if the difference between them is constant, as shown in Fig. 3. This reveals the differences between the presented numerical results and theoretical relations which are smaller than ten percent. This discrepancy is illustrated in Fig. 3, where the theoretical dependence is presented by the dashed line.

For weak flexoelectric properties (e.g., if $\left.e_{11}-e_{33}=10 \mathrm{pC} / \mathrm{m}\right)$ the rotation angle $\Phi$ was proportional to bias voltage. When the difference $e_{11}-e_{33}$ increased, the $\Phi(U)$ dependence became stronger and deviated from proportionality as shown in Fig. 4.

At any maintained voltage, the rotation angle increased with $e_{11}-e_{33}$ in a nonlinear way. This means that the efficiency of the effect saturated when the flexoelectric parameter was enhanced at high voltages. It is worthy to note that the opposite sign of $e_{11}-e_{33}$ led to the opposite rotation of the optical axis. 


\subsubsection{Role of intrinsic pitch}

The intrinsic pitch $p_{0}$ was varied between 0.15 and $0.4 \mu \mathrm{m}$. For a short pitch, the rotation angle was nearly proportional to bias voltage. The coefficient of proportionality increased linearly with intrinsic pitch $p_{0}$ which means that the efficiency of the flexoelectro-optic effect was proportional to $p_{0}$. For a long pitch, nonlinear dependence occurred, as shown in Fig. 5. At the same voltage, the rotation angles were the larger, the longer was the intrinsic pitch.

\subsubsection{Role of thickness and anchoring strength}

For each thickness varying in the range of $1.5-4 \mu \mathrm{m}$ and initial values of other parameters, the rotation angle was proportional to bias voltage. The coefficient of proportionality decreased with thickness so that the efficiency of the flexoelectrooptic effect was inversely proportional to thickness. For the thinnest layer, nonlinear $\Phi(U)$ dependence occurred. Wide regions of bend deformation were observed in thin layers, whereas splay dominated in thick layers.

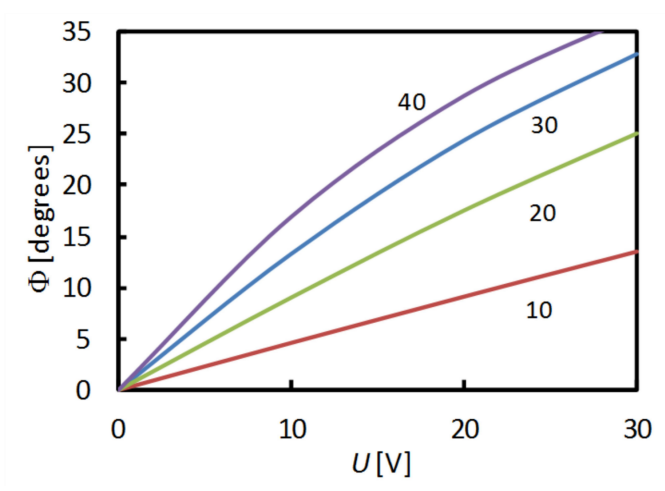

Fig. 4. Rotation angle shown as a function of voltage for different values of $e_{11}-e_{33}$ indicated in $\mathrm{pC} / \mathrm{m}$ at the curves, $e_{33}=0$. Other parameters had their initial values.

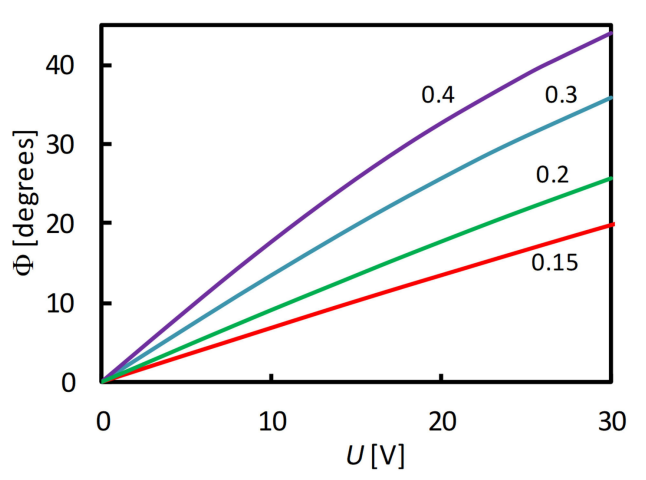

Fig. 5. Rotation angle shown as a function of voltage for various intrinsic pitch lengths indicated in micrometers at the curves. Other parameters had their initial values.

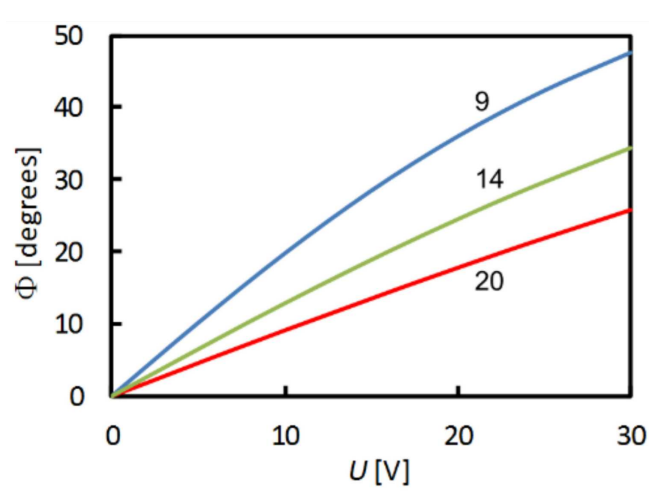

Fig. 6. Rotation angle shown as a function of voltage for different values of $k_{11}+k_{33}$ indicated in $\mathrm{pN}$ at the curves. The following sets of elastic constants $k_{11}, k_{22}, k_{33}$ expressed in pN were used: $(6,1,3)$, $(7,4,7),(8,4,12)$. Other parameters had their initial values.

Interactions between liquid crystal and boundary surfaces, determined by anchoring strength parameters $W_{\theta 1}, W_{\theta 2}, W_{\varphi 1}$ and $W_{\varphi 2}$, were varied from weak, determined by parameters as low as $10^{-6} \mathrm{~J} / \mathrm{m}^{2}$, to strong, reaching $10^{-3} \mathrm{~J} / \mathrm{m}^{2}$. It was found that the anchoring strength parameters did not affect neither the rotation angle nor the pitch.

\subsubsection{Role of elastic constants}

The rotation angle depended on the sum of splay and bend elastic constants $k_{11}+k_{33}$ and not on their particular values. For a high sum of elastic constants (e.g., $k_{11}+k_{33}={ }_{2} 0 \mathrm{pN}$ ) or for low voltages (e.g., $U<10 \mathrm{~V}$ ), the rotation angle was nearly proportional to bias voltage. The efficiency of the flexoelectro-optic effect decreased with the increase of $k_{11}+k_{33}$. For lower values of $k_{11}+k_{33}$ (e.g., $9 \mathrm{pN}$ ), a deviation from proportionality was evident. These features are illustrated in Fig. 6 for three sets of elastic constants. The director distribution depended on a relation between $k_{11}$ and $k_{33}$ even if the sum of them was the same: small $k_{11}$ led to a wide region of bend and a narrow region of splay whereas small $k_{33}$ favoured a wide area of splay and a narrow region of bend. This observation confirms qualitatively the expectations mentioned in [14].

Certain boundary conditions prevent changes of the pitch. The rotation angle is determined by (2) derived under the assumption of constant pitch $p=p_{0}$. A weak influence of $k_{22}$ on the $\Phi(U)$ dependence is also predicted by (2). This feature was confirmed by the present results of calculations. They also showed that proportionality between $\Phi$ and $U$ was satisfied in the wider range of voltage, the smaller was $k_{22}$.

\subsubsection{Role of dielectric anisotropy}

The development of deformations in the presence of dielectric anisotropy depended in a complex way on the sign and magnitude of anisotropy as well as 


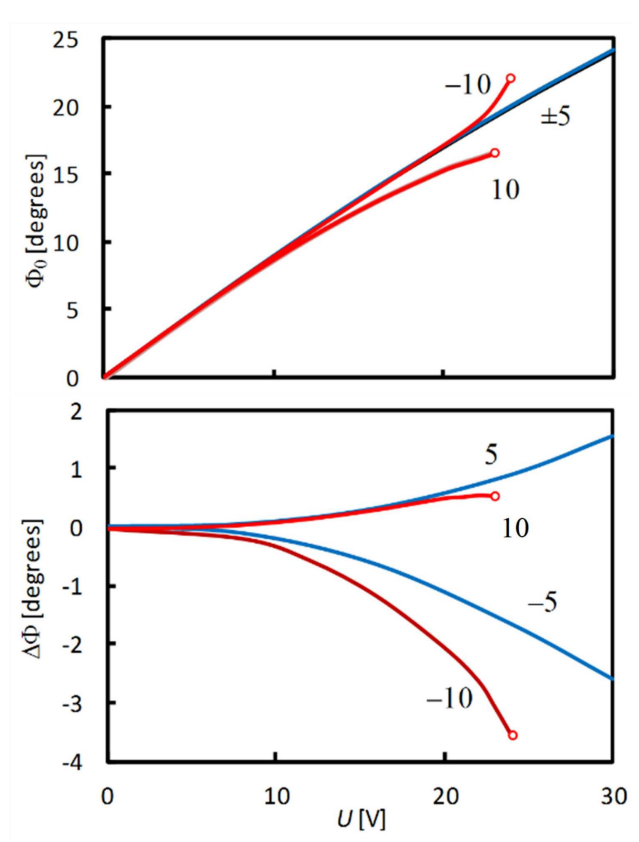

Fig. 7. Average rotation angle $\Phi_{0}$ and the amplitude of sinusoidal variation $\Delta \Phi$ shown as functions of bias voltage. Values of dielectric anisotropy are indicated at the curves. Other parameters had their initial values. Circles denote unwinding of the helical structure.

on the intrinsic pitch $p_{0}$. Dielectric anisotropy gives rise to torque which can lead to deformation or even to unwinding of the helical structure when the layer is subjected to external electric field. Therefore, the zero dielectric anisotropy is required for the proper performance of the flexoelectro-optic effect. Simulations reported in [14] showed that if the cholesteric possessed a significant dielectric anisotropy, the rotation angle $\Phi$ varied sinusoidally along the helix axis. The present calculations confirmed that this variation is described by the function

$$
\Phi(y)=\Phi_{0}+\Delta \Phi \cos \left(\frac{4 \pi y}{p}\right),
$$

where $\Phi_{0}$ and $\Delta \Phi$ depend on $z$ in the vicinity of the boundaries only. The average rotation angle $\Phi_{0}$ increased with voltage. Figure 7 shows that small deformations were independent of dielectric anisotropy in a wider range of voltages. At higher voltages, the $\Phi_{0}(U)$ dependence deviated from proportionality according to suggestions made in [15]. This nonlinearity occurred at lower voltage if $\Delta \varepsilon>0$. Amplitude $\Delta \Phi$ increased with voltage but was rather small in comparison with $\Phi$. The negative anisotropy induced larger amplitude $\Delta \Phi$ and caused a stronger dependence of the rotation angle on $y$ coordinate than the positive anisotropy. Amplitude $\Delta \Phi$ was negative when $\Delta \varepsilon<0$ and positive in the opposite case. In the case of the negative anisotropy, the prevailing part of the layer was dominated by bend, whereas splay dominated in the case of the positive anisotropy.

\subsection{Pitch}

If variations of the pitch were allowed, it decreased with voltage starting from $p_{0}$ at $U=0$, simultaneously with the director rotation (see Fig. 8).

The decrease of the pitch induced by voltage was the stronger, the larger was $p_{0}$, the lower was the sum $k_{11}+k_{33}$, the thinner was the layer (see Fig. 8), and the stronger were the flexoelectric properties (see Fig. 9). The function $p(\Phi)$ had the same form for all thicknesses taken into account. In the case of small deformations occurring when the flexoelectricity was weak, e.g., if $e_{11}-e_{33}=10 \mathrm{pC} / \mathrm{m}$, pitch was proportional to $\cos (\Phi)$ according to (3). This relationship was confirmed by the present computations.

In the presence of dielectric anisotropy, the deformations were more complex than in the case of $\Delta \varepsilon=0$ due to the presence of dielectric torque. Initially, the pitch weakly depended on growing voltage up to some critical value above which it increased rapidly. An undesirable increase of the pitch above the wavelengths of light may lead to the extinction of the flexoelectro-optic effect. Finally, the helical structure became unwound.

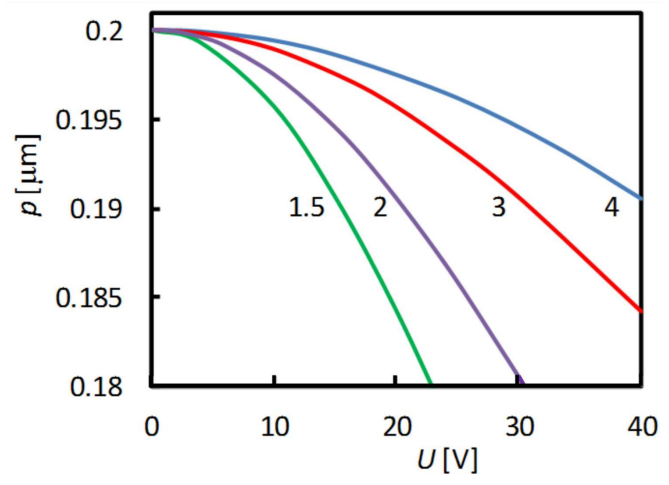

Fig. 8. Pitch shown as a function of voltage for different thicknesses of the layer indicated in micrometers at the curves. Other parameters had their initial values.

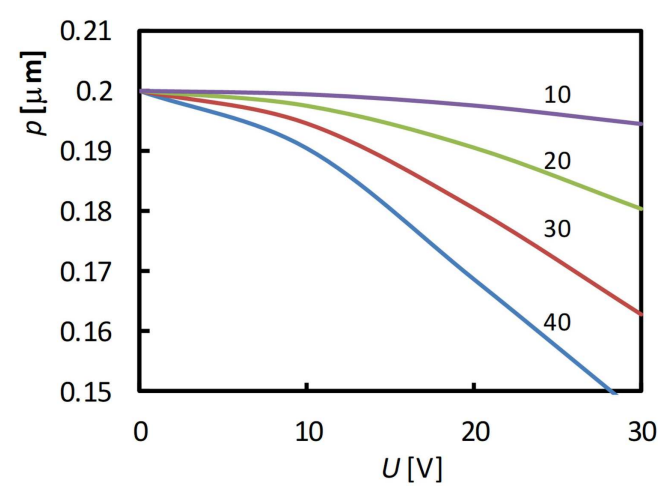

Fig. 9. Pitch shown as a function of voltage for different values of $e_{11}-e_{33}$ indicated in $\mathrm{pC} / \mathrm{m}$ at the curves $e_{33}=0$. Other parameters had their initial values. 


\section{Conclusions}

The director distributions in the ULH structure deformed by bias voltage were determined. The role of parameters important for the behavior of the ULH structure was studied. The linear voltage dependence of the rotation angle of the optic axis was found at low voltages, however deviations from proportionality occurred when the voltage reached sufficiently large values. The voltage dependence of freely deformed pitch was also calculated. The results of simulations confirmed the validity of theoretical formulae (1)-(3) describing the flexoelectrooptic effect. All the curves obtained from numerical computations and presented in Figs. 3-6 and 8, 9 agree with the corresponding curves calculated from theoretical formulae with the accuracy better than ten percent as exemplified in Fig. 3. This concerns the voltage dependence of the rotation angle as well as of the effective pitch occurring at various sets of system parameters. The consequences of non-zero dielectric anisotropy illustrated in Fig. 7. confirmed qualitatively the results reported in [14].

The requirements which should be satisfied in order to obtain great efficiency of the flexoelectrooptic effect were determined. In particular, a chiral material should have the longest pitch which, however, ensures necessary optical properties, the sum of splay and bend elastic constants should be small and the difference between flexoelectric coefficients should be as large as possible. The thinnest layer is preferred, provided that it is fitted to birefringence of the cholesteric in order to satisfy the condition $\sin ^{2}(\pi d \Delta n / \lambda)=1$. The anchoring strength has minor influence if only it ensures a stable ULH structure. The dielectrically compensated cholesteric should be used.

\section{References}

[1] J.S. Patel, R.B. Meyer, Phys. Rev. Lett. 58, 1538 (1987).

[2] S.D. Lee, J.S. Patel, R.B. Meyer, J. App. Phys. 67, 1293 (1990).

[3] P. Rudquist, M. Buivydas, L. Komitov, S.T. Lagerwall, J. Appl. Phys. 76, 7778 (1994).
[4] P. Rudquist, T. Carlsson, L. Komitov, S.T. Lagerwall, Liq. Cryst. 22, 445 (1997).

[5] P. Rudquist, S.T. Lagerwall, Liq. Cryst. 23, 503 (1997).

[6] A. Varanytsia, L. Chien, in: 2015 IEEE Photonics Conf. (IPC), Reston (VA), p. 38.

[7] G. Tan, Y. Lee, F. Gou, H. Chen, Y. Huang Y. Lan, C. Tsai, S. Wu, J. Phys. D: Appl. Phys. 50, 493001 (2017).

[8] Xiuze Wang, J.A. Fells, C. Welch, M.-G. Tamba, G.H. Mehl, S.M. Morris, S.J. Elston, Liq. Cryst. 46, 408 (2018).

[9] M. Buczkowska, Sci. Bull. Lodz Univ. Technol. Phys. 41, 23 (2020).

[10] G. Derfel, M. Buczkowska, Sci. Bull. Lodz Univ. Technol. Phys. 36, 5 (2015).

[11] D. Krzyżański, G. Derfel, Phys. Rev. E 61, 6663 (2000).

[12] M. Buczkowska, G. Derfel, Phys. Rev. E 95, 062705 (2017).

[13] L.M. Blinov, Structure and Properties of Liquid Crystals, Springer, New York 2011.

[14] D.R. Corbett, S.J. Elston, Phys. Rev. E 84, 041706 (2011).

[15] B.J. Outram, S.J. Elston, Liq. Cryst. 40, 1529 (2013). 